\title{
Fatores demográficos e hábitos de vida relacionados com a inatividade física de lazer entre gêneros
}

\section{(1)}

Gender-specific demographic factors and lifestyle habits related to physical inactivity

Suzana Patrícia de SÁ-SILVA ${ }^{1}$

Edna Massae YOKOO

Rosana SALLES-COSTA ${ }^{3}$

RE S U M O

\section{Objetivo}

Analisar a associação entre variáveis sociodemográficas e hábitos de vida com Inatividade Física de Lazer, entre os sexos, em população de baixa renda.

\section{Métodos}

Estudo transversal de base populacional, em Duque de Caxias, Rio de Janeiro (2005). A coleta de dados constou de visita domiciliar e amostra de 1113 adultos. Foram avaliados alguns dados sobre Atividade Física de Lazer, informações sociodemográficas, hábitos de vida (hábito de fumar, consumo de refrigerantes) e medidas antropométricas. Na análise de dados foi empregado o modelo hierárquico, utilizando a regressão de Poisson e a Inatividade Física de Lazer (indivíduos que responderam não praticar nenhum tipo de Atividade Física de Lazer) como desfecho. O primeiro nível do modelo considerou variáveis sociodemográficas e o segundo, o estado nutricional (índice de massa corporal em $\mathrm{kg} / \mathrm{m}^{2}$ (baixo peso $<18$,5; eutrófico $\geq 18,5$ e $<25,0$; sobrepeso $\geq 25,0$ e $<30,0$; e obeso $\geq 30,0$ ), hábitos de vida e consumo alimentar.

\section{Resultados}

A prevalência de Inatividade Física de Lazer foi de 33,0\% (IC95\%: 28,6-37,6) para os homens, e 67,0\% (IC95\%: $62,3-71,3)$ para as mulheres. No modelo final, entre os homens, a Inatividade Física de Lazer foi associada com

${ }^{1}$ Universidade Federal do Rio de Janeiro, Instituto de Nutrição Josué de Castro, Programa de Pós-Graduação em Nutrição. Rio de Janeiro, RJ, Brasil.

2 Universidade Federal Fluminense, Instituto de Saúde da Comunidade, Departamento de Epidemiologia e Bioestatística. Niterói, RJ, Brasil.

${ }^{3}$ Universidade Federal do Rio de Janeiro, Instituto de Nutrição Josué de Castro, Departamento de Nutrição Social e Aplicada. Av. Carlos Chagas Filho, 373, Edifício do Centro de Ciências da Saúde, Bloco J, $2^{\circ}$ andar, Cidade Universitária, 21941-902, Rio de Janeiro, RJ, Brasil. Correspondência para/Correspondence to: R SALLES-COSTA. E-mail: <rosana@nutricao.ufrj.br>. 
634 | SP SÁ-SILVA et al.

excesso de peso (sobrepeso: $\mathrm{RP}=1,50$, obesidade: $\mathrm{RP}=1,50, p$-valor $=0,004$ ) e cor de pele (pretos/pardos: $\mathrm{RP}=0,71$, $p$-valor=0,003). Entre mulheres, a Inatividade Física de Lazer foi associada com escolaridade $(R P=1,15$, $p$-valor=0,019), perímetro de cintura $(R P=0,87, p$-valor $=0,042)$, hábito de fumar $(R P=1,17, p$-valor $=0,003)$ e maior ingestão de refrigerantes ( $\mathrm{RP}=1,22, p$-valor $=0,015)$.

\section{Conclusão}

Os fatores associados com a Inatividade Física de Lazer diferiram entre os gêneros. As mulheres possuíam estilos de vida não saudáveis, ou seja, fumavam, consumiam mais refrigerantes, e eram mais inativas fisicamente.

Termos de indexação: Atividade motora. Estilo de vida. Identidade de gênero.

\section{A B S T R A C T}

\section{Objective}

To identify gender-specific sociodemographic and lifestyle variables associated with leisure-time physical inactivity in a low-income population.

\section{Methods}

This cross-sectional, population-based study conducted in Duque de Caxias, Rio de Janeiro (2005), collected the following data from 1113 adults in home interviews: leisure-time physical activity, sociodemographic information, lifestyle habits (smoking, soda intake), and anthropometric measurements. The hierarchical Poisson regression modeling was used for data analysis setting leisure-time physical inactivity (individuals who did not practice any type of leisure-time physical activity) as outcome. The first level of the model included the sociodemographic variables and the second, lifestyle habits, food intake, and nutritional status according to body mass index $\left(\mathrm{kg} / \mathrm{m}^{2}\right)$ as follows: underweight $<18.5 ; 18.5 \leq$ normal weight $<25.0 ; 25.0 \leq$ overweight $<30.0$; and obese $\geq 30.0$.

\section{Results}

The prevalence of leisure-time physical inactivity was 33.0\% (CI95\%: 28.6-37.6) for men and 67.0\% (C195\%: 62.3-71.3) for women. In the final model, leisure-time physical inactivity in males was associated with overweight (overweight: $P R=1.50$, obesity: $P R=1.50, P=0.004$ ) and skin color (black/brown: $P R=0.71, P=0.003$ ); and in females, with education level $(P R=1.15, p=0.019)$, waist circumference $(P R=0.87, p=0.042)$, smoking $(P R=1.17$, $p=0.003)$, and higher soda intake ( $P R=1.22, p=0.015)$.

\section{Conclusion}

The factors associated with leisure-time physical inactivity vary between genders. The women had unhealthy lifestyles: they smoked, consumed soda, and were more inactive.

Indexing terms: Motor activity. Life style. Gender identity.

\section{N T R O D U ÇÃ O}

Nas últimas décadas, o Brasil vem sofrendo intenso processo de urbanização, com grande mobilização de nossa população para os grandes centros urbanos ${ }^{1}$. As consequências dessa transformação se expressam, entre outras formas, nos padrões de comportamento e qualidade de vida da população, modificando o perfil das doenças e agravos à saúde, principalmente no aumento das doenças crônicas não transmissíveis². Nesse sentido, a construção de Políticas Públicas de Promoção da Saúde ganhou força incentivando, en- tre outros aspectos, modificações na qualidade de vida, como a promoção da atividade física em locais públicos ${ }^{3}$.

Para populações de baixa renda que residem à margem dos centros urbanos, esta tarefa se torna um desafio, dada a influência das desigualdades sociais no estilo de vida destes segmentos. Para exemplificar, estudos revelam que a prática de atividades físicas em países desenvolvidos e emergentes é influenciada negativamente pela baixa renda ${ }^{4}$ e menor escolaridade ${ }^{5}$.

Além dos fatores demográficos, estudos publicados nos últimos cinco anos sugerem que 
fatores relacionados ao estilo de vida como o hábito de fumar $^{6}$ e a realização de dietas saudáveis ${ }^{7}$ influenciam indiretamente no engajamento em atividades físicas no tempo destinado ao lazer. As pesquisas revelam que grupos de menor renda possuem menos chance de praticarem atividade física em clubes ou academias, ficando restrita a prática em lugares públicos ${ }^{9}$, tendendo ao consumo de menor variedade de alimentos, principalmente em relação à ingestão de frutas, verduras e legumes ${ }^{10}$.

Outro ponto a ser destacado se refere às diferenças de gênero no engajamento de Atividades Físicas de Lazer (AFL), uma vez que os homens tendem a ser mais ativos que as mulheres ${ }^{11}$. Para explicar essas diferenças, os autores têm evidenciado que as distintas atribuições nas responsabilidades familiares, bem como na função ocupacional desempenhada por homens e mulheres ${ }^{12}$, além da existência de diferentes padrões sociais e culturais na adesão à prática de esporte ${ }^{4}$ podem ser fatores que explicam o menor engajamento das mulheres nas atividades físicas no tempo destinado ao lazer.

Diante do exposto, o objetivo deste trabalho foi analisar a associação entre fatores sociodemográficos e hábitos de vida com a inatividade física no tempo destinado ao lazer, entre gêneros, em uma população de baixa renda.

\section{M É T O D O S}

Tratou-se de estudo transversal, realizado no período de maio a dezembro de 2005, com amostra complexa por conglomerados de 1125 domicílios selecionados em Campos Elíseos, em Duque de Caxias, Região Metropolitana do Rio de Janeiro. Para determinar o tamanho da amostra, partiu-se da prevalência de $14,5 \%$ de pobreza extrema, de forma que uma amostra composta com 1200 domicílios asseguraria níveis de precisão iguais a $5 \%$. Detalhes do processo amostral e desenho de estudo estão disponíveis em Salles-Costa et al. ${ }^{13}$.
Foram obtidas informações de 1085 domicílios, totalizando perdas de 3,6\% referentes a 29 domicílios (recusas, não localização do domicílio ou do chefe da família após três visitas). Da população amostrada, composta por 1275 adultos (idade entre 19 e 60 anos), foram excluídos 52 indivíduos devido a respostas inconsistentes ou incompletas $(4,0 \%)$ e 110 indivíduos por apresentaram um consumo energético inferior a $500 \mathrm{kcal} /$ dia e superior a $5000 \mathrm{kcal} / \mathrm{dia}(8,6 \%)$, por serem considerados pouco plausíveis. Desta forma, a amostra final avaliada neste estudo foi de 1113 indivíduos, totalizando perdas de $8 \%$.

A coleta de dados constou de visita domiciliar, com entrevista e realização de exame antropométrico. Todos os entrevistadores passaram por treinamento e os questionários utilizados passaram por um pré-teste ${ }^{13}$. Os questionários foram digitados por bolsistas (dupla digitação dos dados).

No momento da entrevista, os participantes foram informados sobre todos os procedimentos realizados, tendo sido apresentado o termo de consentimento para ser assinado, garantindo o anonimato dos resultados obtidos bem como a recusa da realização dos mesmos.

\section{Variáveis de estudo}

As informações foram obtidas por questionário com perguntas sobre a prática de atividade física de lazer, informações sociodemográficas e sobre hábitos de vida, com realização de exame antropométrico.

A atividade física de lazer foi definida como qualquer atividade física praticada para melhorar a saúde e/ou a condição física, ou realizada com o objetivo estético ou de lazer, nos três meses anteriores à aplicação do questionário ${ }^{14}$. Foi avaliada por meio de pergunta fechada em que os indivíduos deveriam informar se praticavam ou não AFL. Neste estudo, não foram consideradas a frequência de realização. Para efeito 
de análise foram considerados inativos fisicamente os indivíduos que responderam não praticar nenhum tipo de AFL, como realizado em outros trabalhos ${ }^{14,15}$.

Para avaliar a influência dos fatores associados à inatividade física de lazer foi utilizado modelo hierárquico, considerando no primeiro nível as variáveis sociodemográficas e no segundo nível, as medidas antropométricas e de hábitos de vida.

As variáveis sociodemográficas utilizadas foram: sexo (feminino/masculino), idade (19-29; 30-49, 50-59 e $\geq 60$ anos), escolaridade ( $\leq 9$ anos - ensino fundamental completo) e mais de 9 anos de estudo - ensino médio completo e universitário); renda familiar mensal per capita (categorizada em tercis), situação conjugal (casado ou união estável ou solteiro/separado), cor da pele (categorizada em branca e preta/parda) e nível de insegurança alimentar das famílias (estimada pela Escala Brasileira de Insegurança Alimentar ${ }^{16}$, segurança alimentar, insegurança leve e inse-gurança moderada e intensa).

Quanto às variáveis de segundo nível, os hábitos de vida consideraram: hábito de fumar (não fumante/fumante), cuidado de crianças menores de três anos (sim/não), risco para compulsão (presença/ausência), realização de dietas (sim/não) e prática de alimentação saudável (sim/não).

Em relação à definição para o hábito de fumar, foram consideradas fumantes aquelas que relataram fumar cigarros diariamente, independente da quantidade consumida. Neste estudo não se consideraram os ex-fumantes.

Para a avaliação do risco de compulsão, o questionário aplicado consistiu de uma adaptação daquele que foi utilizado por Hay ${ }^{17}$ para averiguar a prevalência de transtornos alimentares em uma comunidade australiana. A confiabilidade do mesmo foi analisada em outra pesquisa e foi considerada boa ${ }^{18}$. Neste estudo, foi utilizada a pergunta sobre frequência de compulsão alimentar que consistia de quatro categorias (nenhuma vez, menos que uma vez/semana, uma vez/semana, duas ou mais vezes/semana) nos últimos três meses. Para fins de análise, aqueles que responderam nenhuma vez, foram categorizados como ausência para risco de compulsão e aqueles que relataram a presença do comportamento pelo menos uma vez na semana foram categorizados como presença para o risco de compulsão.

A avaliação da qualidade da alimentação foi realizada por meio de pergunta dicotômica autorreferida obtida a partir de pergunta sobre a percepção de sua alimentação como saudável ou não.

O consumo alimentar da população foi estimado com a aplicação de Questionário de Frequencia Alimentar (QFCA) semiquantitativo, previamente validado por Sichieri \& Everhart ${ }^{19}$, em estudo realizado com uma amostra de funcionários de uma universidade pública do Rio de Janeiro. A lista de alimentos do questionário em questão foi definida a partir dos alimentos mais consumidos pela população brasileira com base no Estudo Nacional de Despesa Familiar (ENDEF) ${ }^{20}$ realizado em 1974-1975, em uma amostra representativa da população, utilizando o método da pesagem de sete dias. Após a validação, a lista contemplou 82 itens alimentares com suas respectivas porções expressas em medidas caseiras, bem como as opções de frequência de consumo (três vezes ou mais/dia, duas a três vezes/dia, uma vez/dia, cinco a seis vezes/semana, duas a quatro vezes/semana, uma vez/semana, uma a três vezes/ mês, nunca ou quase nunca). Mais detalhes do estudo encontram-se disponíveis em Sichieri \& Everhart ${ }^{19}$

A frequência de consumo relatada para frutas e refrigerantes foi convertida em frequência diária, considerando, nas análises, o consumo de refrigerantes em três categorias ( $<1$ vez por dia, $1-2$ vezes por dia, $\geq 3$ vezes por dia) e de Frutas, Legumes e Verduras (FLV) $(<1$ vez ao dia, $1-2$ vezes por dia, 3-4 vezes por dia e $\geq 5$ vezes por dia).

O estado nutricional foi avaliado pelo Índice de Massa Corporal (IMC) e Perímetro de 
Cintura (PC). O peso $(\mathrm{kg})$ foi aferido em balança eletrônica (capacidade de até $150 \mathrm{~kg}$, variação de 100 gramas). A estatura $(\mathrm{cm})$ foi aferida duas vezes com antropômetro portátil (amplitude de $200 \mathrm{~cm}$ e variação de 0,1 centímetros). As médias do (PC) (cm) foram aferidas com o auxílio de fita métrica inextensível (amplitude de 150 centímetros e variação de 0,1 milímetros), de acordo com a padronização de Lohmam et al. ${ }^{21}$. Estimou-se o IMC classificando-o em: baixo peso (IMC $<18,5)$, eutrófico $(\geq 18,5 \mathrm{IMC}<25,0)$, sobrepeso $(\geq 25,0$ $I M C<30,0)$ e obeso (IMC $\geq 30,0)^{22}$. O PC foi classificado de acordo com o risco para doenças cardiovasculares: baixo ou nenhum risco $\mathrm{PC}<80 \mathrm{~cm}$ e $P C<94 \mathrm{~cm}$ e risco elevado ou muito elevado PC $\geq 80 \mathrm{~cm} \mathrm{PC} \geq 94 \mathrm{~cm}$, respectivamente para mulheres e homens ${ }^{22}$.

\section{Análise de dados}

Foram estimadas as proporções, os Intervalos de Confiança a 95\%(IC95\%) e p-valor das variáveis sociodemográficas e hábitos de vida entre homens e mulheres ativos e inativos fisicamente, utilizando o teste Qui-quadrado para comparação entre grupos. Adotou-se modelo hierárquico considerando dois níveis: $1^{\circ}$ nível) variáveis sociodemográficas, e $2^{\circ}$ nível) variáveis relacionadas ao estilo de vida, tendo como desenlace a inatividade física. Por meio de regressão de Poisson, foram testados modelos univariados, para a obtenção da Razão de Prevalência (RP) e seus respectivos IC95\%. Variáveis com $p$-valor $<0,20$ foram incluídas na análise multivariada em cada nível hierárquico, ajustando os modelos em cada nível. Aquelas que se associaram significativamente ao nível de 5\%, com o desfecho em questão, constituíram o modelo hierárquico final (RP ajustada). O banco de dados foi elaborado e digitado no programa CSPro 2.5. Todas as análises apresentaram os valores amostrais referentes à população de estudo, sendo os parâmetros e os respectivos IC95\% estimados para os dados expandidos considerando o efeito do desenho amostral, obtidos no módulo survey data analysis do programa Stata $11^{23}$.
O presente trabalho foi aprovado pelo Comitê de Ética em Pesquisa do Instituto de Medicina Social da Universidade do Estado do Rio de Janeiro (registro 02/2004) em outubro de 2004, e financiado pelo Conselho Nacional de Desenvolvimento Científico e Tecnológico (CNPq) (Ministério de Ciência e Tecnologia/Ministério Extraordinário de Segurança Alimentar e Combate à Fome/CNPq/Centro Tecnologico - Agronegócio 01/2003, Processo n 503139/2003-3).

\section{RESULTADOS}

Dos 1113 participantes, a inatividade física de lazer foi referida por $70,8 \%$ dos adultos, sendo as mulheres mais inativas fisicamente (67,0\% IC95\%: 62,3-71,3) quando comparadas com os homens $(33,0 \%$ IC95\% 28,6-37,6).

Observou-se proporção significativamente maior de homens inativos na faixa entre 30 e 49 anos de idade (homens 45,3\%; mulheres $42,1 \%$ ), com escolaridade superior a 9 anos de estudo (homens 53,0\%; mulheres 35,3\%), que não cuidavam de crianças (homens 95,8\%; mulheres $82,0 \%)$. Entre as mulheres inativas observaram-se proporções significativamente maiores de inatividade física entre aquelas com maior perímetro de cintura (homens 27,5\%; mulheres 48,2\%), com obesidade (homens 12,8\%; mulheres $21,3 \%$ ), que referiram episódios de compulsão alimentar (homens 4,1\%; mulheres 9,2\%), que realizavam dietas (homens 6,4\%; mulheres 15,9\%) e que consumiam até cinco porções ao dia de FLV (homens 74,0\%; mulheres 78,6\%) (Tabela 1).

As análises ajustadas do primeiro nível do modelo hierárquico para os homens mantiveram as associações de menor prevalência de inatividade física no lazer com a cor de pele preta e parda. Análises ajustadas no segundo nível confirmaram a associação entre maior prevalência de inatividade física e excesso de peso. O ajuste também resultou em perda de significância estatística das associações entre inatividade no lazer e perímetro de cintura (Tabela 2). 
638 | SP SA-SILVA et al.

Tabela 1. Características sociodemográficas, hábitos de vida e variáveis antropométricas e atividade física, por sexo, residentes em Campos Elíseos, Duque de Caxias (RJ), 2005.

Continua

\begin{tabular}{|c|c|c|c|c|c|c|c|c|c|c|}
\hline \multirow{3}{*}{ Variáveis } & \multicolumn{5}{|c|}{ Ativos } & \multicolumn{5}{|c|}{ Inativos } \\
\hline & \multicolumn{2}{|c|}{ Homens $(n=156)$} & \multicolumn{2}{|c|}{ Mulheres $(n=172)$} & \multirow{2}{*}{ - $p$-valor ${ }^{*}$} & \multicolumn{2}{|c|}{ Homens ( $n=213)$} & \multicolumn{2}{|c|}{ Mulheres $(n=572)$} & \multirow{2}{*}{$p$-valor } \\
\hline & $\%$ & IC95\% & $\%$ & IC95\% & & $\%$ & IC95\% & $\%$ & IC95\% & \\
\hline \multicolumn{11}{|l|}{ Fatores sociodemográficos } \\
\hline Idade (anos) & & & & & 0,513 & & & & & 0,026 \\
\hline $19-29$ & 24,6 & $16,1-35,8$ & 25,8 & $18,3-35,0$ & & 24,4 & $17,3-33,2$ & 26,0 & $20,3-32,6$ & \\
\hline $30-49$ & 54,4 & $42,8-65,6$ & 44,1 & $33,4-55,3$ & & 45,3 & $36,0-54,9$ & 42,1 & $36,1-48,3$ & \\
\hline $50-59$ & 13,6 & $8,8-20,4$ & 18,5 & $11,3-28,6$ & & 12,3 & $7,9-18,6$ & 21,5 & $16,5-27,7$ & \\
\hline$\geq 60$ & 7,1 & $2,2-20,9$ & 11,5 & $6,5-19,4$ & & 17,8 & $12,3-25,2$ & 10,2 & $6,9-14,7$ & \\
\hline Escolaridade (anos) & & & & & 0,559 & & & & & 0,001 \\
\hline$\leq 9$ anos & 44,0 & $34,5-54,1$ & 48,0 & $37,8-58,3$ & & 46,9 & $36,8-57,3$ & 64,7 & $58,5-70,4$ & \\
\hline$>9$ anos & 55,9 & $45,8-65,5$ & 52,0 & $41,6-62,1$ & & 53,0 & $42,6-63,1$ & 35,2 & $29,5-41,4$ & \\
\hline Cor de pele & & & & & $<0,001$ & & & & & 0,434 \\
\hline Branca & 18,3 & $12,0-26,9$ & 39,4 & $29,4-50,5$ & & 35,6 & $26,2-46,2$ & 31,4 & $25,7-37,8$ & \\
\hline Preto e pardo & 81,7 & $73,1-88,0$ & 60,5 & $49,4-70,6$ & & 64,4 & $53,7-73,7$ & 68,5 & $62,2-74,2$ & \\
\hline Situação conjugal & & & & & 0,405 & & & & & 0,12 \\
\hline Casado & 69,3 & $58,4-78,5$ & 64,6 & $54,7-73,4$ & & 75,6 & $66,3-83,0$ & 67,8 & $61,2-73,8$ & \\
\hline Solteiro ou separado & 30,6 & $21,4-41,5$ & 35,3 & $26,5-45,2$ & & 24,3 & $16,9-33,6$ & 32,1 & $26,1-38,7$ & \\
\hline$\leq R \$ 112,50$ & 18,9 & $10,7-31,2$ & 20,3 & $10,0-36,8$ & & $\begin{array}{l}20,1 \\
42,1\end{array}$ & $\begin{array}{l}14,4-28,9 \\
34,0-50,6\end{array}$ & $\begin{array}{l}30,2 \\
36,8\end{array}$ & $\begin{array}{l}24,1-37,1 \\
30,6-43,6\end{array}$ & \\
\hline $\begin{array}{l}>R \$ 112,50 \text { e }<R \$ 250,00 \\
>R \$ 250,00\end{array}$ & $\begin{array}{l}39,4 \\
41,5\end{array}$ & $\begin{array}{l}27,5-52,7 \\
29,1-55,1\end{array}$ & $\begin{array}{l}39,2 \\
40,3\end{array}$ & $\begin{array}{l}28,0-51,7 \\
29,4-52,4\end{array}$ & & $\begin{array}{l}42,1 \\
37,1\end{array}$ & $\begin{array}{l}34,0-5,6 \\
29,6-45,2\end{array}$ & $\begin{array}{l}30,8 \\
32,8\end{array}$ & $\begin{array}{l}30,6-43,6 \\
25,4-41,3\end{array}$ & \\
\hline Insegurança alimentar & & & & & 0,029 & & & & & 0,081 \\
\hline Segurança alimentar & 58,2 & $45,9-69,5$ & 47,4 & $35,3-59,8$ & & 52,2 & $44,2-60,0$ & 42,4 & $36,2-48,9$ & \\
\hline IA leve & 27,7 & $17,9-40,1$ & 22,7 & $14,8-33,1$ & & 27,1 & $20,4-34,9$ & 33,5 & $27,4-40,2$ & \\
\hline IA moderada e intensa & 14,0 & $9,1-20,8$ & 29,8 & $19,0-43,4$ & & 20,6 & $14,1-29,2$ & 24,0 & $18,5-30,6$ & \\
\hline Cuidar de crianças & & & & & 0,003 & & & & & $<0,001$ \\
\hline Não & 92,8 & $87,5-95,9$ & 80,1 & $69,2-87,7$ & & 95,8 & $92,4-97,7$ & 82,0 & $76,9-86,2$ & \\
\hline $\operatorname{sim}$ & 7,2 & $4,0-12,4$ & 19,8 & $12,2-30,7$ & & 4,1 & $2,2-7,5$ & 17,9 & $13,7-23,0$ & \\
\hline \multicolumn{11}{|l|}{ Estado nutricional } \\
\hline Perímetro da cintura $(\mathrm{cm})$ & & & & & $<0,001$ & & & & & $<0,001$ \\
\hline $\begin{array}{l}<80 \mathrm{~cm} \text { para mulheres }<94 \mathrm{~cm} \\
\text { para homens }\end{array}$ & 84,6 & $75,0-91,0$ & 37,7 & $26,5-50,4$ & & 72,5 & $62,5-80,6$ & 51,8 & $45,1-58,3$ & \\
\hline $\begin{array}{l}\geq 80 \mathrm{~cm} \text { para mulheres } \geq 94 \mathrm{~cm} \\
\text { para homens }\end{array}$ & 15,3 & $8,9-24,9$ & 62,2 & $49,5-73,4$ & & 27,5 & $19,4-37,4$ & 48,2 & $41,6-54,8$ & \\
\hline$I M C\left(k g / m^{2}\right)$ & & & & & 0,004 & & & & & 0,045 \\
\hline Baixo peso & 1,6 & $0,4-5,9$ & 0,4 & $0,1-2,0$ & & 1,4 & $0,3-6,8$ & 4,4 & $2,3-8,2$ & \\
\hline Eutrófico & 60,2 & $50,6-69,1$ & 41,8 & $31,2-53,3$ & & 39,6 & $31,5-48,4$ & 42,0 & $35,9-48,3$ & \\
\hline Sobrepeso & 31,6 & $23,4-41,0$ & 34,3 & $24,1-46,1$ & & 45,9 & $37,3-54,7$ & 32,1 & $27,0-37,8$ & \\
\hline Obesidade & 6,4 & $2,8-14,0$ & 23,3 & $15,0-34,4$ & & 12,8 & $7,0-22,3$ & 21,3 & $16,9-26,4$ & \\
\hline \multicolumn{11}{|l|}{ Hábitos de vida } \\
\hline Fumo & & & & & $<0,001$ & & & & & 0,447 \\
\hline $\operatorname{Sim}$ & 24,3 & $14,1-38,4$ & 5,8 & $2,9-11,1$ & & 16,8 & $10,7-25,6$ & 19,7 & $15,0-25,2$ & \\
\hline Não & 75,6 & $61,5-85,8$ & 94,1 & $88,8-97,0$ & & 83,1 & $74,3-89,2$ & 80,3 & $74,7-84,9$ & \\
\hline Risco para compulsão alimentar & & & & & 0,008 & & & & & 0,041 \\
\hline Não & 95,6 & $90,2-98,1$ & 84,3 & $73,8-91,1$ & & 95,8 & $92,1-97,8$ & 90,8 & $86,0-94,0$ & \\
\hline Sim & 4,3 & $1,8-9,7$ & 15,6 & $8,8-26,1$ & & 4,1 & $2,1-7,8$ & 9,2 & $5,9-13,9$ & \\
\hline
\end{tabular}


Tabela 1. Características sociodemográficas, hábitos de vida e variáveis antropométricas e atividade física, por sexo, residentes em Campos Elíseos, Duque de Caxias (RJ), 2005.

Conclusão

\begin{tabular}{|c|c|c|c|c|c|c|c|c|c|c|}
\hline \multirow{3}{*}{ Variáveis } & \multicolumn{5}{|c|}{ Ativos } & \multicolumn{5}{|c|}{ Inativos } \\
\hline & \multicolumn{2}{|c|}{ Homens $(n=156)$} & \multicolumn{2}{|c|}{ Mulheres $(n=172)$} & \multirow{2}{*}{$p$-valor } & \multicolumn{2}{|c|}{ Homens $(n=213)$} & \multicolumn{2}{|c|}{ Mulheres $(n=572)$} & \multirow{2}{*}{$p$-valor" } \\
\hline & $\%$ & IC95\% & $\%$ & IC95\% & & $\%$ & IC95\% & $\%$ & IC95\% & \\
\hline Alimentação saudável & & & & & 0,192 & & & & & 0,186 \\
\hline Sim & 85,3 & $75,6-91,5$ & 76,6 & $66,3-84,6$ & & 78,3 & $69,5-85,0$ & 72,2 & $65,2-78,3$ & \\
\hline Não & 14,7 & $8,4-24,4$ & 23,3 & $15,3-33,6$ & & 21,7 & $14,9-30,4$ & 27,7 & $21,6-34,7$ & \\
\hline Realização de dieta & & & & & 0,003 & & & & & 0,012 \\
\hline Sim & 11,5 & $6,6-19,4$ & 29,7 & $21,3-39,8$ & & 6,4 & $3,1-12,7$ & 15,9 & $12,0-20,8$ & \\
\hline Não & 88,4 & $80,5-93,4$ & 70,2 & $60,1-78,6$ & & 93,5 & $87,2-96,8$ & 84,0 & $79,1-87,9$ & \\
\hline Consumo FLV (frequência diária) & & & & & 0,258 & & & & & 0,029 \\
\hline$<1$ & 1,2 & $0,2-5,7$ & 2,6 & $0,3-16,9$ & & 0,5 & $0,1-2,4$ & 0,0 & $0,2-0,2$ & \\
\hline $1-2$ & 4,6 & $1,4-14,1$ & 1,5 & $0,5-4,5$ & & 4,7 & $2,2-9,3$ & 8,1 & $4,9-2,2$ & \\
\hline $3-4$ & 19,9 & $12,0-31,2$ & 14,2 & $7,4-24,3$ & & 20,5 & $13,1-30,8$ & 13,0 & $9,8-17,2$ & \\
\hline$\geq 5$ & 74,1 & $62,5-83,0$ & 81,6 & $70,6-89,0$ & & 74,0 & $62,9-82,7$ & 78,6 & $72,5-83,7$ & \\
\hline $\begin{array}{l}\text { Consumo de refrigerantes } \\
\text { (frequência diária) }\end{array}$ & & & & & $<0,001$ & & & & & 0,259 \\
\hline$<1$ & 68,1 & $57,9-76,8$ & 90,8 & $84,8-94,6$ & & 79,3 & $70,8-85,8$ & 85,6 & $80,5-89,6$ & \\
\hline $1-2$ & 29,3 & $20,5-39,9$ & 8,8 & $5,0-14,9$ & & 16,7 & $11,1-24,3$ & 11,7 & $8,0-16,8$ & \\
\hline$\geq 3$ & 2,5 & $0,8-7,4$ & 0,3 & $0,1-1,2$ & & 3,9 & $1,6-8,8$ & 2,5 & $1,2-5,0$ & \\
\hline
\end{tabular}

Nota: *As análises consideraram nível de significância de $p$-valor <0,05. IA: Insegurança Alimentar; IMC: Índice de Massa Corporal; FLV: Frutas, Legumes e Verduras.

Entre as mulheres, o ajuste no primeiro nível do modelo hierárquico manteve as associações de maior prevalência de inatividade física com menor escolaridade. Análises ajustadas do segundo nível, controladas pela escolaridade, corroboraram as associações entre maior prevalência de inatividade física no lazer e hábito de fumar e maior consumo de refrigerantes. Também foram observadas menores prevalências de inatividade entre aquelas com maior perímetro de cintura. O ajuste resultou em perda de significância estatística da associação entre inatividade no lazer e realização de dieta (Tabela 3).

\section{I S C U S S Ã O}

Neste trabalho, identificaram-se diferenças quanto à inatividade física entre homens e mulheres. Os homens negros e pardos eram menos inativos ao passo que aqueles com excesso de peso praticavam menos atividade física. Por outro lado, verificou-se que as mulheres que possuíam estilos de vida não saudáveis, ou seja, que eram fumantes e que possuíam maior ingestão de refrigerantes, eram mais inativas fisicamente. Nesse sentido, esses resultados corroboram os resultados de que, além dos indicadores sociodemográficos ${ }^{6}$, os fatores relacionados ao estilo de vida podem ajudar a elucidar tais questões?

A relação inversa entre tabagismo e atividade física em adultos é bem documentada, possuindo uma associação mais forte nos homens ${ }^{24}$. Kaczynski et al. ${ }^{25}$, em sua revisão sistemática, observou o fumo associado à inatividade física entre as mulheres, como verificado neste estudo. Isso pode ser explicado pelo fato de que adultos sedentários, principalmente as mulheres, podem começar ou continuar a fumar como uma estratégia de controle de peso ${ }^{26}$.

Os achados do presente estudo corroboram resultados de outras pesquisas que observaram a prevalência de inatividade física no lazer associada a um padrão alimentar menos saudável, especialmente entre as mulheres ${ }^{27}$. Nesse sentido, 
640 | SP SÁ-SILVA et al.

Tabela 2. Razão de prevalência (RP) e intervalos de confiança (IC95\%) da associação entre fatores sociodemográficos, hábitos de vida e variáveis antropométricas e inatividade física entre homens residentes em Campos Elíseos, Duque de Caxias (RJ), 2005.

Continua

\begin{tabular}{|c|c|c|c|c|c|c|}
\hline \multirow{2}{*}{ Variáveis de estudo } & \multicolumn{3}{|c|}{ Análise univariada } & \multicolumn{3}{|c|}{ Análise ajustada } \\
\hline & $\mathrm{RP}$ & IC95\% & $p$-valor ${ }^{\star}$ & $\mathrm{RP}$ & IC95\% & $p$-valor ${ }^{*}$ \\
\hline \multicolumn{7}{|l|}{$1^{\circ}$ nível } \\
\hline Idade (anos) & & & 0,137 & & & 0,202 \\
\hline $19-29$ & 1,00 & & & 1,00 & $0,75-1,34$ & \\
\hline $30-49$ & 0,92 & $0,69-1,23$ & & 1,00 & $0,68-1,35$ & \\
\hline $50-59$ & 0,96 & $0,68-1,35$ & & 0,96 & $0,88-2,07$ & \\
\hline$\geq 60$ & 1,33 & $0,91-1,94$ & & 1,35 & & \\
\hline Escolaridade (anos) & & & 0,665 & & & \\
\hline$\leq 9$ anos & 1,05 & $0,83-1,31$ & & & & \\
\hline$>9$ anos & 1,00 & & & & & \\
\hline Cor de pele & & & 0,002 & & & 0,003 \\
\hline Branca & 1,00 & & & 1,00 & & \\
\hline Preto e pardo & 0,71 & $0,58-0,87$ & & 0,71 & $0,58-0,88$ & \\
\hline Situação conjugal & & & 0,259 & & & \\
\hline Casado & 1,14 & $0,87-1,50$ & 0,309 & & & \\
\hline Solteiro ou separado & 1,00 & & & & & \\
\hline Renda (em tercis de Reais) & & & 0,597 & & & \\
\hline$\leq R \$ 112,50$ & 1,08 & $0,76-1,56$ & & & & \\
\hline$>R \$ 112,50$ e $<R \$ 250,00$ & 1,07 & $0,81-1,43$ & & & & \\
\hline$\geq R \$ 250,00$ & 1,00 & & & & & \\
\hline Insegurança alimentar & & & 0,227 & & & \\
\hline Segurança alimentar & 1,00 & & & & & \\
\hline Insegurança alimentar leve & 1,03 & $0,79-1,35$ & & & & \\
\hline Insegurança alimentar moderada e intensa & 1,20 & $0,92-1,58$ & & & & \\
\hline Cuidar de crianças & & & 0,151 & & & 0,222 \\
\hline Não & 1,00 & & & 1,00 & & \\
\hline $\operatorname{Sim}$ & 0,74 & $0,49-1,11$ & & 0,78 & $0,52-1,16$ & \\
\hline \multicolumn{7}{|l|}{$2^{\circ}$ nível } \\
\hline Perímetro da cintura $(\mathrm{cm})$ & & & 0,050 & & & 0,946 \\
\hline$<94$ & 1,00 & & & 1,00 & & \\
\hline$\geq 94$ & 1,29 & $1,00-1,67$ & & 1,04 & $0,75-1,45$ & \\
\hline IMC $\left(\mathrm{kg} / \mathrm{m}^{2}\right)$ & & & $<0,001$ & & & 0,005 \\
\hline Baixo peso & 1,16 & $0,50-2,68$ & & 1,06 & $0,43-2,62$ & \\
\hline Eutrófico & 1,00 & & & 1,00 & & \\
\hline Sobrepeso & 1,52 & $1,21-1,92$ & & 1,49 & $1,17-1,89$ & \\
\hline Obesidade & 1,55 & $1,10-2,19$ & & 1,47 & $0,94-2,28$ & \\
\hline Fumo & & & 0,317 & & & \\
\hline $\operatorname{sim}$ & 0,80 & $0,51-1,24$ & & & & \\
\hline Não & 1,00 & & & & & \\
\hline Risco para compulsão alimentar & & & 0,887 & & & \\
\hline Não & 1,00 & & & & & \\
\hline $\operatorname{Sim}$ & 1,03 & $0,68-1,55$ & & & & \\
\hline Alimentação saudável & & & 0,175 & & & 0,284 \\
\hline $\operatorname{Sim}$ & 1,00 & & & 1,00 & & \\
\hline Não & 1,20 & $0,91-1,57$ & & 1,17 & $0,93-1,46$ & \\
\hline
\end{tabular}


Tabela 2. Razão de prevalência (RP) e intervalos de confiança (IC95\%) da associação entre fatores sociodemográficos, hábitos de vida e variáveis antropométricas e inatividade física entre homens residentes em Campos Elíseos, Duque de Caxias (RJ), 2005.

\begin{tabular}{|c|c|c|c|c|c|c|}
\hline \multirow{2}{*}{ Variáveis de estudo } & \multicolumn{3}{|c|}{ Análise univariada } & \multicolumn{3}{|c|}{ Análise ajustada } \\
\hline & $\mathrm{RP}$ & IC95\% & $p$-valor ${ }^{\star}$ & $\mathrm{RP}$ & IC95\% & $p$-valor \\
\hline Realização de dieta & & & 0,269 & & & \\
\hline Sim & 1,00 & & & & & \\
\hline Não & 1,34 & $0,79-2,26$ & & & & \\
\hline Consumo FLV (frequência diária) & & & 0,991 & & & \\
\hline$<1$ & 0,46 & $0,06-3,21$ & & & & \\
\hline $1-2$ & 1,08 & $0,72-1,61$ & & & & \\
\hline $3-4$ & 1,01 & $0,71-1,44$ & & & & \\
\hline$\geq 5$ & 1,00 & & & & & \\
\hline Consumo de refrigerantes (frequência diária) & & & 0,133 & & & 0,161 \\
\hline$<1$ & 1,00 & & & 1,00 & & \\
\hline $1-2$ & 0,69 & $0,49-0,99$ & & 0,71 & $0,51-0,99$ & \\
\hline$\geq 3$ & 1,03 & $0,62-1,70$ & & 1,25 & $0,74-2,11$ & \\
\hline
\end{tabular}

Nota: *Considerando o nível de significância $p$-valor $\leq 0,20$ para inclusão nos modelos de regressão hierárquicos. IMC: Índice de Massa Corporal; FLV: Frutas, Legumes e Verduras.

os autores têm sugerido que a presença de hábitos alimentares mais saudáveis é mais frequente entre praticantes de atividades físicas, como um auxílio à manutenção de uma alimentação adequada, expressa pelo maior consumo de vegetais e frutas e menor de refrigerantes?

Somado às dificuldades na prática de AFL, a precariedade ao acesso a uma alimentação saudável, rica em frutas, verduras e legumes e pobre em refrigerantes, como preconizado pela Política Nacional de Alimentação e Nutrição ${ }^{28}$, deve ser ressaltada. Indivíduos de menor renda vivem em condições de saúde e de alimentação comprometidas devido ao comprometimento da renda para manter uma alimentação saudável e de qualidade ${ }^{29}$. Esse fato foi observado entre as mulheres mais pobres deste estudo, uma vez que, além de serem inativas, também possuíam uma alimentação inadequada.

Estas questões são importantes para o desenvolvimento de programas de intervenção que enfatizam a prática de atividade física e de estilo de vida saudáveis ${ }^{30}$. O desenvolvimento de políticas públicas que considerem fatores de risco modificáveis, como o tabagismo, inatividade física e alimentação inadequada, podem ser utéis para o controle das doenças crônicas não transmissíveis, que atualmente representa um problema de saúde pública ${ }^{31}$.

Ao avaliar a Estratégia Global para Alimentação, Atividade Física e Saúde implementada em 2004 pela Organização Mundial de Saúde ${ }^{32}$ observamos recomendações de que os indivíduos se envolvam em níveis adequados de atividade física, aproveitando os espaços domésticos e públicos próximos às suas casas para exercitarem-se. Nesse sentido, cabe salientar que, em ambos os casos, o tema não aborda a questão da ausência de espaços físicos para a prática esportiva e diferenças de gênero.

Quando avaliamos os espaços públicos para a prática de exercícios no Brasil, a presença de campos de futebol são as ferramentas públicas mais frequentes para a prática de exercícios físicos, o que enfatiza os esportes coletivos e atividades tipicamente masculinas ${ }^{33}$. São escassos os lugares que dispõem de espaços para caminhadas ou para ginástica, exercícios preferencialmente escolhidos pelas mulheres ${ }^{15}$. Assim, as mulheres que pertencem a um segmento social menos favorecido, sem condições financeiras para as práticas de AFL em clubes ou academias, as chances de se engajarem em AFL são ainda menores ${ }^{9}$. 
642 SP SÁ-SILVA et al.

Tabela 3. Razão de prevalência (RP) e intervalos de confiança (IC95\%) da associação entre fatores sociodemográficos, hábitos de vida e variáveis antropométricas e a inatividade física entre mulheres residentes em Campos Elíseos, Duque de Caxias (RJ), 2005.

Continua

\begin{tabular}{|c|c|c|c|c|c|c|}
\hline \multirow{2}{*}{ Variáveis de estudo } & \multicolumn{3}{|c|}{ Análise univariada } & \multicolumn{3}{|c|}{ Análise ajustada } \\
\hline & $\mathrm{RP}$ & IC95\% & $p$-valor ${ }^{*}$ & $\mathrm{RP}$ & IC95\% & $p$-valor ${ }^{*}$ \\
\hline \multicolumn{7}{|l|}{$1^{\circ}$ nivel } \\
\hline Idade (anos) & & & 0,979 & & & \\
\hline $19-29$ & 1,00 & & & & & \\
\hline $30-49$ & 0,98 & $0,86-1,12$ & & & & \\
\hline $50-59$ & 1,02 & $0,88-1,20$ & & & & \\
\hline$\geq 60$ & 0,97 & $0,80-1,17$ & & & & \\
\hline Escolaridade (anos) & & & 0,008 & & & 0,019 \\
\hline$\leq 9$ anos & 1,16 & $1,04-1,30$ & & 1,15 & $1,02-1,29$ & \\
\hline$>9$ anos & 1,00 & & & 1,00 & & \\
\hline Cor de pele & & & 0,203 & & & \\
\hline Branca & 1,00 & & & & & \\
\hline Preto e pardo & 1,07 & $0,95-1,21$ & & & & \\
\hline Situação conjugal & & & 0,561 & & & \\
\hline Casado & 1,03 & $0,92-1,14$ & & & & \\
\hline Solteiro ou separado & 1,00 & & & & & \\
\hline Renda (em tercis de Reais) & & & 0,182 & & & 0,277 \\
\hline$\leq \mathrm{R} \$ 112,50$ & 1,12 & $0,94-1,33$ & & 1,10 & $0,92-1,31$ & \\
\hline$>R \$ 112,50$ e $<R \$ 250,00$ & 1,03 & $0,89-1,19$ & & 1,02 & $0,88-1,19$ & \\
\hline$\geq R \$ 250,00$ & 1,00 & & & 1,00 & & \\
\hline Insegurança alimentar & & & 0,951 & & & \\
\hline Segurança alimentar & 1,00 & & & & & \\
\hline Insegurança alimentar leve & 1,09 & $0,98-1,22$ & & & & \\
\hline Insegurança alimentar moderada e intensa & 0,97 & $0,82-1,15$ & & & & \\
\hline Cuidar de crianças & & & 0,774 & & & \\
\hline Não & 1,00 & & & & & \\
\hline Sim & 0,97 & $0,84-1,13$ & & & & \\
\hline \multicolumn{7}{|l|}{$2^{\circ}$ nivel } \\
\hline Perímetro da cintura $(\mathrm{cm})$ & & & 0,009 & & & 0,042 \\
\hline$<80$ & 1,00 & & & 1,00 & & \\
\hline$\geq 80$ & 0,85 & $0,75-0,95$ & & 0,87 & $0,77-0,99$ & \\
\hline$I M C\left(k g / m^{2}\right)$ & & & 0,309 & & & \\
\hline Baixo peso & 1,20 & $1,06-1,35$ & & & & \\
\hline Eutrófico & 1,00 & & & & & \\
\hline Sobrepeso & 0,97 & $0,85-1,10$ & & & & \\
\hline Obesidade & 0,93 & $0,80-1,07$ & & & & \\
\hline Fumo & & & 0,001 & & & 0,003 \\
\hline Sim & 1,19 & $1,07-1,31$ & & 1,17 & $1,06-1,30$ & \\
\hline Não & 1,00 & & & 1,00 & & \\
\hline Risco para compulsão alimentar & & & 0,209 & & & \\
\hline Não & 1,00 & & & & & \\
\hline Sim & 0,86 & $0,68-1,08$ & & & & \\
\hline Alimentação saudável & & & 0,627 & & & \\
\hline Sim & 1,00 & & & & & \\
\hline Não & 1,03 & $0,91-1,16$ & & & & \\
\hline
\end{tabular}


Tabela 3. Razão de prevalência (RP) e intervalos de confiança (IC95\%) da associação entre fatores sociodemográficos, hábitos de vida e variáveis antropométricas e a inatividade física entre mulheres residentes em Campos Elíseos, Duque de Caxias (RJ), 2005.

\begin{tabular}{|c|c|c|c|c|c|c|}
\hline \multirow{2}{*}{ Variáveis de estudo } & \multicolumn{3}{|c|}{ Análise univariada } & \multicolumn{3}{|c|}{ Análise ajustada } \\
\hline & $\mathrm{RP}$ & IC95\% & $p$-valor ${ }^{*}$ & $\mathrm{RP}$ & IC95\% & $p$-valor ${ }^{*}$ \\
\hline Realização de dieta & & & 0,014 & & & 0,086 \\
\hline Sim & 1,00 & & & 1,00 & & \\
\hline Não & 1,24 & $1,04-1,49$ & & 1,17 & $0,97-1,40$ & \\
\hline Consumo FLV (frequência diária) & & & 0,673 & & & \\
\hline$<1$ & 0,15 & $0,01-1,16$ & & & & \\
\hline $1-2$ & 1,19 & $1,09-1,32$ & & & & \\
\hline $3-4$ & 0,97 & $0,84-1,13$ & & & & \\
\hline$\geq 5$ & 1,00 & & & & & \\
\hline Consumo de refrigerantes (frequência diária) & & & 0,004 & & & 0,015 \\
\hline$<1$ & 1,00 & & & 1,00 & & \\
\hline $1-2$ & 1,10 & $0,97-1,25$ & & 1,10 & $0,96-1,25$ & \\
\hline$\geq 3$ & 1,26 & $1,12-1,41$ & & 1,22 & $1,09-1,36$ & \\
\hline
\end{tabular}

Nota: ${ }^{*}$ Considerando o nível de significância $p$-valor $\leq 0,20$ para inclusão nos modelos de regressão hierárquicos. IMC: Índice de Massa Corporal; FLV: Frutas, Legumes e Verduras.

Aliado a isso, quando se observa os dados do IBGE, constata-se que o município de Duque de Caxias possui apenas $31 \%$ de estabelecimentos de saúde públicos, enquanto a média nacional é de $55 \%$. Em 2010, a taxa de analfabetismo das pessoas de 10 anos ou mais era de $4,7 \%^{1}$. Dessa forma, essa situação pode dificultar o acesso a informações acerca de hábitos de vida saudáveis, como manter uma alimentação adequada, não fumar e praticar atividades físicas.

Em relação à inatividade física de mulheres em áreas de baixa renda, a coexistência do número inadequado de creches públicas nos centros urbanos pode ser outro fator que contribui para esse comportamento. O presente trabalho revelou a presença elevada de domicílios com crianças, o que tende a dificultar a prática de AFL entre as mulheres que não têm com quem deixar seus filhos.

Uma das limitações deste trabalho se refere à menor proporção de homens avaliados, quando comparados com o total de mulheres, decorrente das perdas diferenciais na amostra de adultos selecionados durante a coleta de dados. Durante a coleta de dados, observou-se que os homens se ausentavam mais dos domicílios, mesmo após três visitas previamente agendadas.
Outra possível limitação se refere à natureza transversal das análises, já que não se pode inferir causalidade dos resultados (causalidade reversa) uma vez que a exposição e o desfecho foram coletados simultaneamente ${ }^{34}$.

\section{CON CLUS ÃO}

Neste trabalho observou-se que os fatores associados com a inatividade física diferiram entre homens e mulheres. Estas possuíam estilos de vida não saudáveis, ou seja, eram fumantes, ingeriam menos frutas, verduras, legumes e consumiam mais refrigerantes além de serem mais inativas no lazer.

Os resultados observados podem contribuir para o desenvolvimento de programas de intervenção que enfatizam a prática de atividade física no tempo destinado ao lazer, assim como para o desenvolvimento de estilo de vida mais saudáveis respeitando as distinções de sexo, além das desigualdades sociais.

\section{COLABORADORES}

SP SÁ-SILVA participou da concepção, análise de dados e redação do artigo. EM YOKOO participou 
da concepção e redação do artigo. R SALLES-COSTA responsável pela elaboração do projeto, coordenação do estudo e de todas as etapas da elaboração do artigo e da análise de dados.

\section{REFER Ê N CIAS}

1. Instituto Brasileiro de Geografia e Estatística. Censo 2010. Brasil: IBGE; 2010

2. Brasil. Ministério da Saúde. A vigilância, o controle e a prevenção das doenças crônicas não-transmissíveis: DCNT no contexto do Sistema Único de Saúde brasileiro. Brasília: Ministério da Saúde; 2005.

3. Malta DC, Castro AM, Gosch CS, Cruz DKA, Bressan A, Nogueira JD, et al. A política nacional de promoção da saúde e a agenda da atividade física no contexto do SUS. Rev Bras Ativ Fís Saúde. 2008; 13(1):24-7.

4. Azevedo $M R$, Araújo $C L$, Reichert FF, Siqueira FV, Silva MC, Hallal PC. Gender differences in leisuretime physical activity. Int J Public Health. 2007; 52(1):8-15. doi: 10.1007/s00038-006-5062-1.

5. Meseguer CM, Galan I, Herruzo R, Zorrilla B, Rodriguez-Artalejo F. Leisure-time physical activity in a Southern European Mediterranean country: Adherence to recommendations and determining factors. Rev Esp Cardiol. 2009; 62(10):1125-33. doi: 10.1016/S1885-5857(09)73327-4.

6. Olinto MT, Willett WC, Gigante DP, Victora CG. Sociodemographic and lifestyle characteristics in relation to dietary patterns among young Brazilian adults. Public Health Nutr. 2010; 14(1):150-9. doi: $10.1017 /$ S136898001000162X

7. Rintala $M$, Lyytikainen $A$, Leskinen $T$, Alen $M$, Pietilainen $\mathrm{KH}$, Kaprio J, et al. Leisure-time physical activity and nutrition: A twin study. Public Health Nutr. 2011; 14(5):846-52. doi: 10.1017/ S136898001000090X

8. Sodergren M, McNaughton SA, Salmon J, Ball K, Crawford D. Associations between fruit and vegetable intake, leisure-time physical activity, sitting time and self-rated health among older adults: Cross-sectional data from the WELL study. BMC Public Health. 2012; 12(1):551. doi: 10.1186/ 1471-2458-12-551.

9. Salvador EP, Florindo AA, Reis RS, Costa EF. Perception of the environment and leisure-time physical activity in the elderly. Rev Saúde Pública. 2009; 43(6):972-80. doi: 10.1590/S0034-8910 2009005000082.

10. Homenko DR, Morin PC, Eimicke JP, Teresi JA, Weinstock RS. Food insecurity and food choices in rural older adults with diabetes receiving nutrition education via telemedicine. J Nutr Educ Behav. 2010; 42(6):404-9. doi: 10.1016/j.jneb.2009.0 8.001 .

11. Nogueira D, Faerstein E, Rugani I, Chor D, Lopes CS, Werneck GL. Does leisure-time physical activity in early adulthood predict later physical activity? Pro-Saude Study. Rev Bras Epidemiol. 2009; 12:3-9. doi: 10.1590/S1415-790X2009000100001.

12. Santos R, Silva P, Santos P, Ribeiro JC, Mota J. Physical activity and perceived environmental attributes in a sample of Portuguese adults: Results from the Azorean physical activity and health study. Prev Med. 2008; 47(1):83-8. doi: 10.1016/j.ypmed. 2008.02.027.

13. Salles-Costa R, Pereira RA, Vasconcellos MTL, Veiga GV, Marins VMR, Jardim BC, et al. Associação entre fatores socioeconômicos e insegurança alimentar: estudo de base populacional na Região Metropolitana do Rio de Janeiro, Brasil. Rev Nutr. 2008; 21(Supl):99s-109s.

14. Salles-Costa R, Heilborn ML, Werneck GL, Faerstein E, Lopes CS. Gênero e prática de atividade física de lazer. Cad Saúde Pública. 2003; 19 (Supl 2):S325-33. doi: 10.1590/S0102-311X2003000800014.

15. Sa Silva SP, Sandre-Pereira G, Salles-Costa R. Fatores sociodemográficos e atividade física de lazer entre homens e mulheres de Duque de Caxias/RJ. Ciênc Saúde Colet. 2011; 16(11):4491-501. doi: 10.15 90/S1413-81232011001200022.

16. Perez-Escamilla R, Segall-Correa AM, Kurdian ML, Sampaio MMF, Marin-Leon L, Panigassi G. An adapted version of the U.S. Department of Agriculture Food Insecurity module is a valid tool for assessing household food insecurity in Campinas, Brazil. J Nutr. 2004; 134(8):1923-8.

17. Hay P. The epidemiology of eating disorder behaviors: An Australian community-based survey. Int J Eating Disord. 1998; 23(4):371-82. doi: 10.10 02/(SICI)1098-108X(199805)23:4<371::AID-EAT4> 3.0.CO;2-F [pii].

18. Ferreira JES, Veiga GV. Confiabilidade (teste-reteste) de um questionário simplificado para triagem de adolescentes com comportamentos de risco para transtornos alimentares em estudos epidemiológicos. Rev Bras Epidemiol. 2008; 11(3):393-401. doi: 10.1590 .

19. Sichieri R, Everhart JE. Validity of a Brazilian food frequency questionnaire against dietary recalls and estimated energy intake. Nutr Res. 1998; 18(10): 1649-59. doi: 10.1016/S0271-5317(98)00151-1.

20. Instituto Brasileiro de Geografia e Estatística. Estudo nacional de despesa familiar. Brasil: IBGE; 1974.

21. Lohman TG, Roche AF, Martorell R. Antropometric standardization reference manual. Champaing (IL): Human Kinetics Books; 1988. 
22. Centers for Disease Control and Prevention. Basics about overweight and obesity. Geneve: CDC; 2002.

23. Stata Statistical Software: Release 11. College Station (TX): StataCorp LP; 2009.

24. Charilaou M, Karekla M, Constantinou M, Price S. Relationship between physical activity and type of smoking behavior among adolescents and young adults in Cyprus. Nicotine Tob Res. 2009; 11(8): 969-76. doi: 10.1093/ntr/ntp096.

25. Kaczynski AT, Manske SR, Mannell RC, Grewal K. Smoking and physical activity: A systematic review. Am J Health Behav. 2008; 32(1):93-110. doi: 10.5555/ajhb.2008.32.1.93.

26. Gonseth S, Jacot-Sadowski I, Diethelm PA, Barras $\mathrm{V}$, Cornuz J. The tobacco industry's past role in weight control related to smoking. Eur J Public Health. 2012; 22(2):234-7. doi: 10.1093/eurpub/ckr 023.

27. Camoes M, Lopes C. Dietary intake and different types of physical activity: Full-day energy expenditure, occupational and leisure-time. Public Health Nutr. 2007; 6:1-8. doi: 10.1017/\$136898 0007001309.

28. Brasil. Ministério da Saúde. Política nacional de alimentação e nutrição. Brasília: Ministério da Saúde; 2012.
29. Canesqui AM, Garcia RWD. Antropologia e nutrição: um diálogo possível. Rio de Janeiro: Fiocruz; 2005.

30. Arao T, Oida Y, Maruyama C, Mutou T, Sawada S, Matsuzuki $\mathrm{H}$, et al. Impact of lifestyle intervention on physical activity and diet of Japanese workers. Prev Med. 2007; 45(2-3):146-52. doi: 10.1016/ j.ypmed.2007.05.004.

31. Yusuf S, Pitt B. A lifetime of prevention: The case of heart failure. Circulation. 2002; 10:106(24): 2997-8. doi: 10.1161/01.CIR.0000046804.138 47.5D.

32. Waxman A. Why a global strategy on diet, physical activity and health? World Rev Nutr Diet. 2005; 95:162-6. doi: 10.1159/000088302.

33. Teixeira MS, Moore FE. A mulher e o esporte: a experiência dos municípios do Rio de Janeiro e de São Paulo: relatório final. Rio de Janeiro: Instituto Noos; 2008.

34. Medronho RA, Bloch KV, Luiz RR, Werneck GL. Epidemiologia. 2a ed. Atheneu; 2008.

Recebido em: 3/5/2013

Versão final em: 4/10/2013

Aprovado em: 16/10/2013 
